\title{
MICA-BBVA: a factor model of economic and financial indicators for short-term GDP forecasting
}

\author{
Máximo Camacho • Rafael Doménech
}

Received: 14 August 2010 / Accepted: 29 August 2011 / Published online: 24 September 2011 (C) The Author(s) 2011. This article is published with open access at SpringerLink.com

\begin{abstract}
In this paper we extend the Stock and Watson's (Leading economic indicators, new approaches and forecasting records, 1991) single-index dynamic factor model in an econometric framework that has the advantage of combining information from real and financial indicators published at different frequencies and delays with respect to the period to which they refer. We find that the common factor reflects the behavior of the Spanish business cycle well. We also show that financial indicators are useful for forecasting output growth, particularly when certain financial variables lead the common factor. Finally, we provide a simulated real-time exercise and prove that the model is a very useful tool for the short-term analysis of the Spanish Economy.
\end{abstract}

Keywords Business cycles · Output growth $\cdot$ Short-term forecasting

JEL Classification $\quad \mathrm{E} 32 \cdot \mathrm{C} 22 \cdot \mathrm{E} 27$

We thank M. Cardoso, I. Chacón, R. Falbo, J. F. Izquierdo, R. Méndez, J. Rodriguez-Vález and C. Ulloa, the editor and two anonymous referees for their helpful comments and suggestions. M. Camacho and R. Doménech would like to thank CICYT for its support through grants ECO2010-19830,

ECO2008-04669 and ECO2011-29050, respectively. All the remaining errors are our own responsibility.

M. Camacho $(\varangle)$

Facultad de Economía y Empresa,

Departamento de Métodos Cuantitativos para la Economía,

Universidad de Murcia, 30100 Murcia, Spain

e-mail: mcamacho@um.es

R. Doménech

BBVA Research, Madrid, Spain

R. Doménech

University of Valencia, Valencia, Spain 


\section{Introduction}

In the two decades leading up to 2007, industrialized economies faced one of the most stable periods of economic activity the world has ever seen. As some authors have documented, the sharp decrease in the volatility of macroeconomic variables was unprecedented, and the period began to be widely known as "The Great Moderation". The view at the time was that macroeconomic policy had advanced to the point of guaranteeing smooth business cycles, considerably decreasing the probability of tail risks associated with sharp reductions in output and employment. However, this buoyant view was put into question when a financial crisis erupted during the second half of 2007, leading to the sharpest and most generalized fall in output since the Great Depression. In this state of affairs, governments and central banks embarked on aggressive fiscal and monetary policies in order to avoid the breakdown of the financial system, substitute private expenditure with public spending and limit the fall of economic activity. However, decisions about the size and timing of these policies were made on real-time estimates of GDP growth, which is observed with some delay. Doubts about how reliable, comprehensive and up-to-date the available data is in giving information about the state of the economy could introduce additional uncertainty to policymakers. Therefore, in this context it seems of utmost importance to be able to accurately assess the short-term economic developments of GDP, in order for policymakers to have a timely and adequate response to these movements.

Despite the efforts by national statistics agencies during the last decades to mitigate the problems associated with the delays in data publication, the fact is that the first official estimates of GDP growth for a particular quarter are published several weeks after the quarter has finished. For example, in Spain, the flash estimates of GDP by INE are now available about six weeks after the end of the quarter. Nonetheless, forecasters, financial institutions and policy makers in need of monitoring economic activity on a day-to-day basis must rely on monthly, or even weekly, indicators which come up within the quarter, such as production and consumption indicators, labour market variables or financial data. However, mixing quarterly and shorter frequencies in real time is not straightforward due to missing data within quarters. In addition, data sets usually exhibit ragged ends due to the unsynchronized publication of data, which must be incorporated in the forecasting models as soon as the variables are released.

This paper describes a method to deal with all of the shortcomings previously discussed. Following the proposal of Camacho and Perez Quiros (2010), the econometric framework described here is an extension of the Stock and Watson (1991) single-index dynamic factor model, which decomposes the joint dynamic of GDP and a selected set of available indicators into a common latent factor and some idiosyncratic components. Particularly, our model has the advantage of combining information from indicators with different frequencies that are published with different delays with respect to the period to which they refer. The estimate is carried out by maximum likelihood and the common factor extraction, and the filling in of missing data is assessed using the Kalman filter. The proposed model is called MICA-BBVA since it is a factor Model of economic and financial Indicators which is used to monitor the Current development of the economic Activity by Banco Bilbao Vizcaya Argentaria (BBVA). 
In the context of forecasting Spanish economic activity, this paper is closely related to Camacho and Sancho (2003) and to Camacho and Perez Quiros (2011), who propose alternative methods for providing forecasts by using large-scale and small-scale factor models, respectively (see also Cuevas and Quilis 2009). ${ }^{1}$ However, three distinctive features characterize the specification and the model evaluation process proposed in this paper. The first contribution to the previous literature is the use of financial time series as leading indicators of output growth, in a factor model that accounts for asynchronous co-movements between the financial and the real activity indicators. According to the excellent review of the literature by Wheelock and Wohar (2009), it still remains an open question whether financial series help in forecasting growth. Although many studies find that financial indicators are useful for forecasting output growth at about one-year horizons, they also acknowledge that the ability of some financial series (such as the slope of the yield curve) to forecast output growth has declined since the mid-1980s.

In the context of the Spanish economy, Camacho and Perez Quiros (2011) find that financial series do not provide valuable information to develop GDP growth forecasts from a dynamic factor model apart from that contained in hard and soft indicators. However, they reach this result after relating financial series with contemporaneous movements in the common factor. Not surprisingly, our results suggest that the correlation between future economic activity and the slope of the yield curve (the interest rate of the 10-year Spanish debt minus the 3-month euribor) is positive and significant, while the lead is estimated to be about 9 months. Furthermore, we find that the lower the real credit growth and the higher the financial stress on financial markets, the lower the rate of GDP growth. In addition, by means of a Monte Carlo experiment we measure the extent to which the appropriate consideration of financial indicators leads improves its forecasting accuracy.

As a second distinctive feature, we use enlarged historical time series of the Spanish GDP recently published by INE. In contrast to the research by Camacho and Perez Quiros (2011), where the sample started in the mid nineties, we allow our data set to date back to the early eighties. Accordingly, the business cycle indicator is available from that date, and the forecasting evaluation includes other significant expansions and slowdowns, apart from the current 2008-2009 recession, reinforcing the empirical reliability of our results. In addition, we design Monte Carlo simulations which support the view that the lower the persistence of the common factor the greater the gains of enlarging the sample. Finally, we show that the estimated common factor is able to capture the Spanish business cycle dynamics early with a high degree of precision.

The third distinctive feature of our analysis has to do with the forecasting simulation design. As in Camacho and Sancho (2003), the forecasts are carried out in a recursive way: with every new vintage, the model is re-estimated and the forecasts for different horizons are computed. However, their out-of-sample study did not take into account the lag of synchronicity in data publication that characterizes the real-time data flow and it may lead to unrealistically high forecasting performance. Typically, surveys and financial variables are published right at the end of the respective month while real

\footnotetext{
1 See Alvarez et al. (2011) for an extensive discussion of pros and cons of forecasting with small scale versus large-scale dynamic factor models.
} 
activity indicators are published with a delay of up to 2 months. ${ }^{2}$ To overcome this drawback of standard out-of-sample forecasting analyses, we evaluate the forecasting ability of the model by developing a pseudo real-time exercise. ${ }^{3}$ We construct the data vintages used to compute the recursive forecasts by mimicking the pattern of the actual chronological order of the data releases. In the empirical analysis, we show that our model would have accurately forecasted the Spanish GDP over the past 20 years. The model yields significant forecasting improvements over benchmark predictions computed from models that are only based on standard autoregressive specifications.

The structure of this paper is as follows. Section 2 outlines the model, shows how to mix frequencies, states the time series dynamic properties, and describes the state space representation. Section 3 presents the empirical analysis and the main results of the paper. Section 4 concludes and proposes several future lines of research.

\section{The model}

\subsection{Mixing frequencies}

Let us assume that the level of quarterly GDP, $Y_{t}^{*}$, can be decomposed as the sum of three unobservable monthly values $Y_{t}, Y_{t-1}, Y_{t-2}$. For instance, the GDP for the third quarter of a given year is the sum of the GDP corresponding to the 3 months of the third quarter, $Y_{I I I}^{*}=Y_{09}+Y_{08}+Y_{07}$. Among others, Mariano and Murasawa (2003) have shown that if the sample mean of the three within quarter monthly observations can be well approximated by the geometric mean, then the quarterly growth rates can be decomposed as weighted averages of monthly growth rates. In particular, the quarterly growth rate, $y_{t}^{*}$, is approximated by the weighted sum of five monthly growth rates $y_{t}$ :

$$
y_{t}^{*}=\frac{1}{3} y_{t}+\frac{2}{3} y_{t-1}+y_{t-2}+\frac{2}{3} y_{t-3}+\frac{1}{3} y_{t-4} .
$$

It is worth mentioning that in a related paper, Aruoba et al. (2009) avoid the approximation of sample averages by geometric averages but at the cost of assuming that the trend of the time series can be well described by deterministic trends. However, these authors have recently acknowledged that the benefits of moving to the geometric approximation of flow data exceeded the costs of assuming deterministic trends and, in the current versions of their index of business cycle conditions, they use the geometric approximation as well. ${ }^{4}$

\subsection{Dynamic properties}

The model follows the lines proposed by Camacho and Perez Quiros (2010), which is an extension of the dynamic factor model suggested by Stock and Watson (1991).

\footnotetext{
2 To facilitate the analysis, following Giannone et al. (2008) financial data enter into the model as monthly averages since the bulk of information compiled from the indicators is monthly.

3 See Giannone et al. (2008) for further details of pseudo real-time analyses.

4 Proietti and Moauro (2006) also avoid this approximation but at the cost of moving to non-linear models.
} 
Let us assume that the variables introduced in the model are somehow related to the overall economic conditions. We consider a single-index model such that each variable can be written as the sum of two stochastic components: a common component, $x_{t}$, which represents the overall business cycle conditions, and an idiosyncratic component, which refers to the particular dynamics of the series. ${ }^{5}$ The underlying business cycle conditions are assumed to evolve with $A R(p 1)$ dynamics

$$
x_{t}=\rho_{1} x_{t-1}+\cdots+\rho_{p 1} x_{t-p 1}+e_{t},
$$

where $e_{t} \sim i N\left(0, \sigma_{e}^{2}\right)$.

Apart from constructing an index of the business cycle conditions, we are interested in computing accurate short-term forecasts of GDP growth rates. To compute these forecasts, we start by assuming that the evolution of the 3-month growth rates depends linearly on $x_{t}$ and on their idiosyncratic dynamics, $u_{t}^{y}$, which evolve as an $A R(p 2)$

$$
\begin{aligned}
& y_{t}=\beta_{y} x_{t}+u_{t}^{y}, \\
& u_{t}^{y}=d_{1}^{y} u_{t-1}^{y}+\cdots+d_{p 2}^{y} u_{t-p 2}^{y}+\varepsilon_{t}^{y},
\end{aligned}
$$

where $\varepsilon_{t}^{y} \sim i N\left(0, \sigma_{y}^{2}\right)$. In addition, the idiosyncratic dynamics of the $k$ monthly indicators can be expressed in terms of autoregressive processes of $p 3$ orders:

$$
\begin{aligned}
z_{t}^{i} & =\beta_{i} x_{t}+u_{t}^{i}, \\
u_{t}^{i} & =d_{1}^{i} u_{t-q}^{i}+\cdots+d_{p 3}^{i} u_{t-p 3}^{i}+\varepsilon_{t}^{i},
\end{aligned}
$$

where $\varepsilon_{t}^{i} \sim i N\left(0, \sigma_{i}^{2}\right)$. Finally, we assume that all the shocks $e_{t}, \varepsilon_{t}^{y}$, and $\varepsilon_{t}^{i}$, are mutually uncorrelated in cross-section and time-series dimensions.

\subsection{State space representation}

To start, we assume that all the variables included in the model were observed at monthly frequencies for all periods. The exact form of the expressions relating the variables as entered into the model, the common factor, and the idiosyncratic components, depends on the nature of the time series and the transformation that they receive prior to be used in the model.

With respect to GDP quarterly growth rates, one can use expressions (1), and (3), to examine its relationship with the idiosyncratic component and the common factor, which becomes

$$
\begin{aligned}
y_{t}^{*}= & \beta_{y}\left(\frac{1}{3} x_{t}+\frac{2}{3} x_{t-1}+x_{t-2}+\frac{2}{3} x_{t-3}+\frac{1}{3} x_{t-4}\right) \\
& +\left(\frac{1}{3} u_{t}^{y}+\frac{2}{3} u_{t-1}^{y}+u_{t-2}^{y}+\frac{2}{3} u_{t-3}^{y}+\frac{1}{3} u_{t-4}^{y}\right) .
\end{aligned}
$$

\footnotetext{
5 The single-index specification adopted in this paper is a very useful simplifying assumption but it does not preclude us from using additional factors such as financial or price factors. However, enlarging the model to consider multiple factors is out of the scope of this paper and is left for further research.
} 
Hard and soft indicators are treated as follows. To avoid the noisy signals that characterize hard indicators, they are used in annual growth rates. Soft indicators are used in levels since by construction their levels exhibit high correlation with the annual growth rate of their reference series. Calling $Z_{i}^{*}$ the annual growth rates of hard or the level of soft variables, the relationship between the indicators, the common factor, and their idiosyncratic components is

$$
Z_{i t}^{*}=\beta_{i} \sum_{j=0}^{11} x_{t-j}+u_{t}^{i},
$$

with $i=1,2, \ldots, k 1$.

Given its novelty in this type of analysis, the treatment of financial indicators in the dynamic factor model deserves special attention. Wheelock and Wohar (2009) point out that financial variables are usually leading rather than coincident indicators of the economic activity. They argue that the higher the slope of the yield curve, the higher the growth rate which is expected to be observed in future quarters. According to their proposal, we establish the relationship between the level (in the case of term spreads and the slope of the yield curve) or annual growth rate (in the case of total credit) of the financial indicator, $Z_{f t}^{*}$, and the $h$-period future values of the common factor, which represents the overall state of the economy, as follows:

$$
Z_{f t}^{*}=\beta_{f} \sum_{j=0}^{11} x_{t+h-j}+u_{t}^{f}
$$

The model described in (7)-(9) can easily be written in state space representation. Without loss of generalization, we assume that our model contains only GDP, one non-financial indicator and one financial indicator, which are collected in the vector $Y_{t}=\left(y_{t}^{*}, Z_{i t}^{*}, Z_{f t}^{*}\right)^{\prime}{ }^{6}$ For simplicity sake, we also assume that $p 1=p 2=p 3=1$, and that the lead for the financial indicator is $h=1$. In this case, the observation equation, $Y_{t}=Z \alpha_{t}$, is

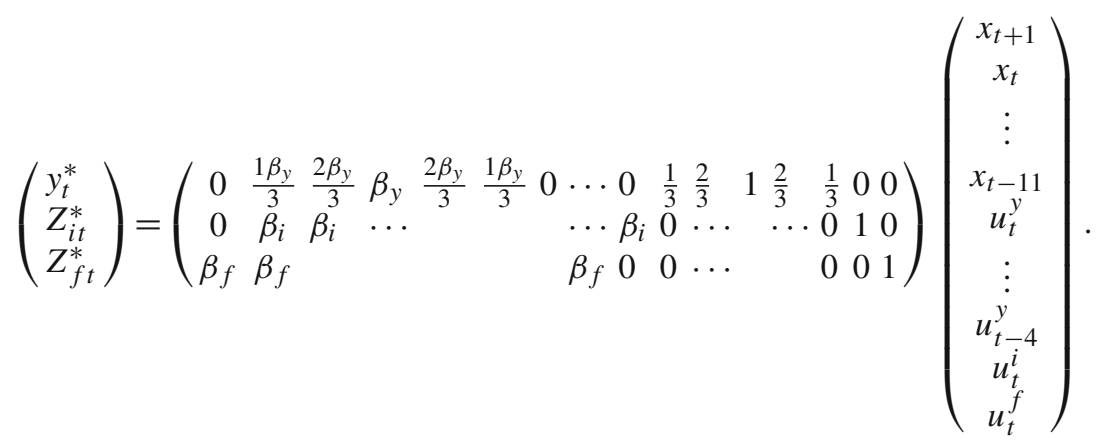

\footnotetext{
6 Allowing the model to account for more indicators is straightforward. In addition, we will look for the appropriate lead in the empirical application.
} 
It is worth noting that the model assumes contemporaneous correlation between nonfinancial indicators and the state of the economy, whereas for financial variables, the correlation is imposed between current values of the indicators and future values of the common factor.

The transition equation, $\alpha_{t}=T \alpha_{t-1}+\eta_{t}$, is

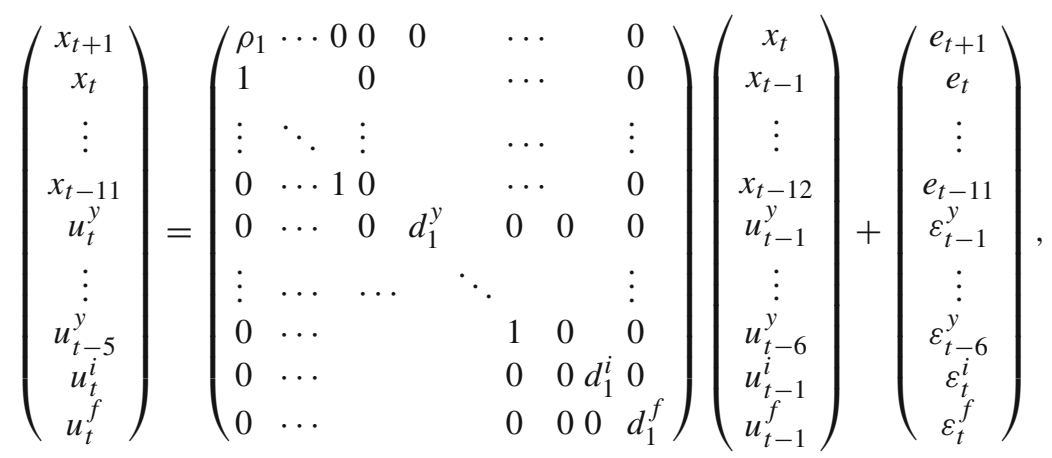

where $\eta_{t} \sim i N(0, Q)$ and $Q=\operatorname{diag}\left(\sigma_{e}^{2}, 0, \ldots, 0, \sigma_{y}^{2}, 0 \ldots 0, \sigma_{i}^{2}, \sigma_{f}^{2}\right)$.

\subsection{Estimation and signal extraction}

The Kalman filter can be used to estimate model's parameters and to infer unobserved components and missing observations. Starting the algorithm with initial values $\alpha_{0 \mid 0}$ and $P_{0 \mid 0}$, the prediction equations are

$$
\begin{aligned}
\alpha_{t+1 \mid t} & =T \alpha_{t \mid t}, \\
P_{t+1 \mid t} & =T P_{t \mid t} T \prime+Q,
\end{aligned}
$$

where $\alpha_{t+1 \mid t}$ is the estimate of the state vector at $t+1$ conditional on the information available at $t$ and $P_{t+1 \mid t}$ is its corresponding covariance matrix. They can be used to compute prediction errors and the their covariance matrix

$$
\begin{aligned}
v_{t+1 \mid t} & =Y_{t}-Z \alpha_{t+1 \mid t}, \\
F_{t+1 \mid t} & =Z P_{t \mid t} Z \prime,
\end{aligned}
$$

which can be used to evaluate the log likelihood function

$$
l_{t}=-\frac{1}{2}\left[\ln \left(2 \pi\left|F_{t \mid t}\right|\right)+v_{t+1 \mid t}^{\prime}\left(F_{t \mid t}\right)^{-1} v_{t+1 \mid t}\right] .
$$

Finally, the state vector and its covariance matrix are updated

$$
\begin{aligned}
& \alpha_{t+1 \mid t+1}=\alpha_{t+1 \mid t}+P_{t+1 \mid t} Z^{\prime}\left(F_{t+1 \mid t}\right)^{-1} v_{t+1 \mid t} \\
& P_{t+1 \mid t+1}=P_{t+1 \mid t}-P_{t+1 \mid t} Z^{\prime}\left(F_{t+1 \mid t}\right)^{-1} Z P_{t+1 \mid t} .
\end{aligned}
$$


So far, we have assumed that all the variables included in the model are always available at monthly frequencies for all time periods. However, this assumption is quite unrealistic when using dynamic factor models to compute forecasts in real time for two reasons. The first reason has to do with mixing quarterly and monthly frequencies, since quarterly data is only observed in the third month of the respective quarter. The second reason has to do with the flow of real-time data. Some indicators are shorter in sample length since they have been constructed only recently. In addition, the publication lag of the indicators is also different. Hard indicators are published with a delay of up to 2 months, soft indicators are usually published at the end of the respective month, and some financial indicators are published daily.

As described in Mariano and Murasawa (2003), the system of equations remains valid with missing data after a subtle transformation. These authors propose replacing the missing observations with random draws $\vartheta_{t}$, whose distribution cannot depend on the parameter space that characterizes the Kalman filter. ${ }^{7}$ To understand the effects of the replacements in the Kalman filter, let us assume that the first element of $Y_{t}$ is missing. Let us call $Y_{t}^{+}$the vector of observations $Y_{t}$ where the first element is replaced by a random draw $\vartheta_{t}$. Since $Y_{t}^{+}$does not contain missing observations, one can use it in the Kalman filter to compute the new likelihood $l_{t}^{+}$which is equivalent to $l_{t}$ up to a scale. In this case, the measurement equation should be replaced by $Y_{t}^{+}=Z_{t}^{+} \alpha_{t}+\omega_{t}$, where $Z_{t}^{+}$is obtained by replacing the first row $Z_{t}$ with zeroes, and $\omega_{t}$ is a vector whose first element is $\vartheta_{t}$ and zeroes elsewhere. Accordingly, the first row will be skipped from the updating in the Kalman recursion.

Given its importance for forecasting, one should note that if all the elements of $Y_{t}$ are missing, the updating equations are skipped, and the Kalman filter will provide the user of the model with time series forecasts for all the series of the model.

\subsection{Monte Carlo analysis}

We stated in the Introduction that the two main contributions of this paper to the previous literature were the use of enlarged time series and the use of financial data as leading indicators of output growth. In this section, we evaluate the relative gains of these contributions by means of a small Monte Carlo experiment. To isolate the effects on forecasting of these two features from other characteristics of the dynamic factor model, the experiment has omitted mixing frequencies and ragged ends. In the simulations, the autoregressive processes are always of order one, all the loading factors are equal to one, and the number of time series is three.

The simulated time series evolve according to expression (3), where the common component follows expression (2), with $\sigma_{e}^{2}=1$, and the idiosyncratic components follow (4), where $\sigma_{i}^{2}=0.5$, and $i=1,2,3$. To evaluate the relative gains of forecasting with dynamic factor models in periods of economic turbulences (low persistence of the common factor) with respect to forecasting in peaceful periods (high persistence

\footnotetext{
$\left.\overline{7 \text { We assume that } \vartheta_{t} \sim N\left(0, \sigma_{\vartheta}^{2}\right.}\right)$ for convenience but replacements by constants would also be valid.
} 
Table 1 Monte Carlo simulations

\begin{tabular}{llll}
\hline & $\rho_{1}=0.8$ & $\rho_{1}=0.5$ & $\rho_{1}=0.1$ \\
\hline $\begin{array}{l}\text { Enlarging the sample size } \\
N=50\end{array}$ & & & \\
$\quad d_{1}^{i}=0.1$ & 0.81 & 0.75 & 0.62 \\
$d_{1}^{i}=0.5$ & 0.89 & 0.80 & 0.72 \\
$N=200$ & 0.72 & 0.66 & 0.53 \\
$\quad d_{1}^{i}=0.1$ & 0.78 & 0.65 & 0.51 \\
$d_{1}^{i}=0.5$ & & & \\
Using financial indicators & & 0.57 & 0.47 \\
MICA/MICA* & & \\
$\quad d_{1}^{i}=0.5$ & 0.64 & 0.57 & 0.55 \\
MICA/MICA** & & & \\
$d_{1}^{i}=0.5$ & 0.58 & & \\
\hline
\end{tabular}

The first block of entries show the MSE of forecasting with MICA over the MSE of forecasting with a random walk. The second block of entries show the MSE of forecasting with MICA over the MSE of forecasting with a MICA that erroneously assume contemporaneous correlation of financial series and the common factor $\left(\mathrm{MICA}^{*}\right)$ and over the MSE of forecasting with a MICA that does not use financial indicators (MICA $\left.{ }^{* *}\right) . N$ is the sample size, $\rho_{1}$ and $d_{1}^{i}$ are the autocorrelation parameters of common and idiosyncratic components

of the common factor whose evolution is quite flat around its historical average), the persistence of the factor is changed from $\rho_{1}=0.8$ to $\rho_{1}=0.5$ and $\rho_{1}=0.1 .^{8}$

In each simulation $j$, of a total amount of $J$ simulations, the time series are generated with $N+1$ observations. All the models are estimated by using only the first $N$ observations while the last figure of the first generated time series, $y_{1 N+1}^{j}$, is used to evaluate the accuracy of the models to compute the $J$ resulting one-period ahead outof-sample forecasts. The forecasting accuracy of the models used in this experiment is analyzed in Table 1. The entries of this table are computed from the mean-squared forecast errors (MSE), which are the averaged deviations of the models predictions from $y_{1 N+1}^{j}$ over the $J=1,000$ simulations.

The top panel of Table 1 examines the relative performance of the dynamic factor model with respect to a benchmark of non-forecastability when the sample size increases from 50 to 200 . In this case, the experiment assumes synchronous dynamics between the three generated time series and their common factor $(h=0)$. Finally, we leave the persistence of the idiosyncratic components to change from $d_{1}^{i}=0.1$ to $d_{1}^{i}=0.5$, where $i=1,2,3$. Overall, the relative MSE rarely exceed 0.80 , pointing out the good forecasting performance of the dynamic factor model over the benchmark. Notably, enlarging the sample from $N=50$ to $N=200$ yields large improvements of the dynamic factor model over the benchmark that range from 10 to $30 \%$. It is worth noting that when the evolution of the factor is quite flat around its historical average (which is accounted for by the case of high persistence of the factor) the relative reductions obtained by enlarging the sample diminish considerably. For example,

\footnotetext{
8 The results for different parameter's values, which are qualitatively similar to those presented in the paper, are available from the authors upon request.
} 
enlarging the sample when $\rho_{1}=0.8$ leads to relative MSE reductions of about $10 \%$ only. However, the relative gains of the dynamic factor model are particularly relevant when the common factor is not very persistent. For concreteness, the reduction of the relative MSE is about $30 \%$ when $\rho_{1}=0.1$ and $d_{1}^{i}=0.5$. This result suggests that using short samples, where the economic activity fluctuates around its historical averages, could largely diminish the actual relatively good performance of dynamic factor models over simpler benchmarks.

The relative gains of correctly specifying the dynamic factor model in the presence of financial indicators is examined in the bottom panel of Table 1. In this case, each simulation assumes that while the dynamics of the first two generated variables and the dynamics of the common factor are synchronized, the third generated variable lead the common factor in one period. Accordingly, the first two variables can be considered as "coincident indicators of economic activity" while the third variable can be considered as a "financial leading indicator". In each simulation, the forecasts $y_{1 N+1}^{j}$ are computed from three alternative dynamic factor models: the first forecast is computed from a dynamic factor model that correctly specifies the lead of the financial leading indicator, the second forecast is computed from a model that erroneously assumes that all the three variables are coincident indicators, and the third forecast is computed from a model that does not use the leading indicator. The MSE obtained from these three alternative forecasts are labelled in Table 1 as MICA, MICA* and MICA $^{* *}$, respectively. According to the table, the correct specification of the dynamic factor model leads to MSE reductions between 40 and 50\%. These results suggest that accounting for the leading behaviour of financial indicator is clearly advisable when using dynamic factor models to forecast GDP growth rates.

\section{Empirical results}

\subsection{Preliminary analysis of data}

The data set used to obtain all the results of this paper cover the period from January 1980 to December 2009. From a list of potential business cycle indicators, we have chosen to include those that verify certain properties in the model. First, they must exhibit high statistical correlation with the GDP growth rate. Second, they should be promptly available at monthly frequency in the sample considered. Third, they must be relevant in the model from both theoretical and empirical points of view and must show explanatory power in terms of the estimated model.

After a careful process of selection, which is described below, the indicators finally included in our model are listed in Table 2 and can be classified as hard, soft and financial indicators. The hard indicators are measures of economic activity such as real GDP, real wage income, electricity consumption, social security affiliates, registered unemployment, and real credit card spending (deflated with Consumer Price Index) from Servired. Typically, hard indicators are published with a reporting lag between 1 and 1.5 months. Soft indicators are based on opinion surveys concerning households (consumer confidence) and manufacturing (industry confidence) and are released on a timely basis. 
Table 2 Final variables included in the model

\begin{tabular}{|c|c|c|c|c|c|}
\hline & Series & $\begin{array}{l}\text { Effective } \\
\text { Sample }\end{array}$ & Source & $\begin{array}{l}\text { Publication } \\
\text { delay } \\
\text { (months) }\end{array}$ & $\begin{array}{l}\text { Data } \\
\text { transformation }\end{array}$ \\
\hline 1 & Real GDP (GDP) & 2Q80-3Q09 & INE & 1.5 & SA, QGR \\
\hline 2 & $\begin{array}{l}\text { Real credit card } \\
\text { spending (CCS) }\end{array}$ & Feb01-Nov09 & $\begin{array}{l}\text { BBVA based on } \\
\text { Servired \& INE }\end{array}$ & 0 & SA, AGR \\
\hline 3 & $\begin{array}{l}\text { Consumer } \\
\text { confidence (CC) }\end{array}$ & Jun86-Nov09 & $\begin{array}{l}\text { European } \\
\text { Commission }\end{array}$ & 0 & $\mathrm{SA}, \mathrm{L}$ \\
\hline 4 & Real wage income (RWI) & Jan81-Oct09 & BBVA based on MEF & 1.5 & AGR \\
\hline 5 & Electricity demand (EC) & Jan81-Oct09 & MEF & 1.5 & SA, TA, AGR \\
\hline 6 & Industry confidence (IC) & Jan87-Nov09 & $\begin{array}{l}\text { European } \\
\text { Commission }\end{array}$ & 0 & $\mathrm{SA}, \mathrm{L}$ \\
\hline 7 & $\begin{array}{l}\text { Registered } \\
\text { unemployment } \\
\text { (U) }\end{array}$ & Jan81-Oct09 & $\begin{array}{c}\text { BBVA ERD based } \\
\text { on INEM (MEI) }\end{array}$ & 1 & SA, AGR \\
\hline 8 & $\begin{array}{l}\text { Social security } \\
\text { affiliation (SSA) }\end{array}$ & Jan81-Oct09 & MEI & 1 & SA, AGR \\
\hline 9 & $\begin{array}{l}\text { Real credit to the } \\
\text { private sector } \\
\text { (RCPS) }\end{array}$ & Jan81-Sep09 & $\begin{array}{l}\text { Bank of Spain and } \\
\text { INE }\end{array}$ & 2 & SA, AGR \\
\hline 10 & $\begin{array}{l}\text { Mortgage rate minus } \\
\text { 12-month Euribor } \\
\text { (MR12E) }\end{array}$ & Jan89-Sep09 & $\begin{array}{l}\text { Bank of Spain \& } \\
\text { Thomson Financial }\end{array}$ & 2 & $\mathrm{~L}$ \\
\hline 11 & $\begin{array}{l}\text { Slope of the yield } \\
\text { curve (SLOPE) }\end{array}$ & Nov87-Nov09 & Thomson Financial & 0 & $\mathrm{~L}$ \\
\hline 12 & $\begin{array}{l}\text { Mortgage rate minus } \\
\text { 12-month Treasury } \\
\text { bill rate } \\
\text { (MR12TBR) }\end{array}$ & Jan81-Sep09 & $\begin{array}{l}\text { Bank of Spain \& } \\
\text { Thomson Financial }\end{array}$ & 2 & $\mathrm{~L}$ \\
\hline
\end{tabular}

QGR, AGR and L mean quarterly growth rates, annual growth rates and levels

$S A$ Seasonally adjusted, TA temperature adjusted, INE National Statistics Institute, MEF Ministry of economy and finance, $M E I$ Ministry of employment and immigration

Among the financial indicators, we include four variables. First, the slope of the yield curve (10-year Spanish bond rate minus 3-month Euribor) which is available with no reporting lags. Second, two measures of financial markets tensions, such as the average mortgage rate minus the 12-month Euribor and the average mortgage rate minus the 12-month Treasury bill rate. The last two financial indicators exhibit a reporting lag of 2 months. Finally, we include the annual growth rate of real credit to the private sector (deflated using core inflation), which is published with a delay of 2 months. It is worth pointing out that although some of the financial variables are published with considerable delay, they are included in the model since they have proven to forecast GDP growth.

All the variables are seasonally adjusted, including calendar adjustments and outlier detection and correction. ${ }^{9}$ GDP enters in the model as its quarterly growth rate, hard indicators and total credit enter in annual growth rates, and confidence and finan-

\footnotetext{
9 Non-seasonally adjusted series from official sources have been treated with Tramo-Seats (see Gomez and Maravall 1996).
} 
Table 3 Cross correlations between the variables in the model

\begin{tabular}{lllllllllllll}
\hline$j$ & GDP & CCS & CC & RWI & EC & IC & U & SSA & RCPS & MR12S & SLOPE & MR12TBR \\
\hline 0.00 & 1.00 & 0.86 & 0.74 & 0.59 & 0.66 & $0.72-0.68$ & 0.74 & 0.45 & -0.28 & 0.19 & -0.37 \\
1.00 & 0.72 & 0.85 & 0.74 & 0.46 & 0.59 & $0.61-0.60$ & 0.65 & 0.37 & -0.21 & 0.30 & -0.32 \\
2.00 & 0.59 & 0.83 & 0.63 & 0.32 & 0.50 & $0.45-0.52$ & 0.54 & 0.29 & -0.13 & 0.34 & -0.24 \\
3.00 & 0.57 & 0.78 & 0.48 & 0.17 & 0.42 & $0.34-0.44$ & 0.43 & 0.19 & -0.08 & 0.36 & -0.24 \\
4.00 & 0.31 & 0.78 & 0.36 & 0.15 & 0.31 & $0.26-0.33$ & 0.34 & 0.07 & -0.03 & 0.35 & -0.25 \\
5.00 & 0.23 & 0.75 & 0.28 & 0.11 & 0.21 & $0.18-0.24$ & 0.25 & -0.03 & 0.02 & 0.33 & -0.16 \\
6.00 & 0.21 & 0.69 & 0.23 & 0.10 & 0.11 & $0.15-0.15$ & 0.16 & -0.12 & 0.11 & 0.32 & -0.08 \\
\hline
\end{tabular}

See Table 2 for a description of the variables. GDP refers to q-o-q rate of growth. Each row shows the correlation between GDP at $t+j$ and the corresponding variable at $t$

cial indicators enter in levels, therefore, with no transformation. Before estimating the model, the variables are standardized to have a zero mean and a variance equal to one. ${ }^{10}$

In Table 3, we present the correlation between the final variables included in our model dated at $t$ and quarterly GDP growth rates dated at $t+j$, with $j=0,1,2, \ldots, 6$. As expected, all variables show a positive contemporaneous $(j=0)$ correlation with quarterly GDP growth, except unemployment $(U)$, the mortgage rate minus 12-month Euribor (MRI2E), and the mortgage rate minus 12-month Treasury bill rate $(M R 12 T B R)$. Noticeably, for all the nonfinancial indicators the magnitude of the dynamic correlation is maximized in the case of their contemporaneous correlations. However, one important exception is the correlations displayed in the case of the yield curve. Although the contemporaneous correlation between GDP growth and the slope of the yield curve is small (0.19), it is much higher and statistically significant for lags of the slope between 2 to 9 quarters. ${ }^{11}$ This result suggests that nonfinancial indicators should be modelled with contemporaneous dynamics with the common factor (Eq. 5) while financial indicators should be modelled as leading the factor by $h$ periods (Eq. 8), with $h$ endogenously determined by the model.

\subsection{In-sample analysis}

The problem of selecting indicators from a universe of potentially available time series is still an open question in empirical studies regarding factor models. Although the number of time series available in a timely manner increases continuously as the information technology improves, the empirical research is usually restricted to a "reduced" amount of "standard" indicators. In the case of the US, empirical studies usually deal with slight transformations of the set of about two hundred time series initially used by Stock and Watson (2002). In the case of European data, the sets of indicators usually employed in empirical research are subtle modifications of the set of about eighty variables initially proposed by Angelini et al. (2011).

\footnotetext{
10 Therefore, final forecasts are recursively computed by multiplying initial forecasts of the model by the standard deviation, and then adding the mean. Then, means and standard deviations are recursively recomputed.

11 To save space, significance tests are excluded but they are available from the authors upon request.
} 
In this paper, the selection of Spanish indicators to be used in the dynamic factor model follows the recommendations suggested by Camacho and Perez Quiros (2010, 2011). ${ }^{12}$ Following Stock and Watson (1991), they propose to start with a model that includes measures of industrial production (industry confidence), employment (social security affiliates), and personal income (real wage income), enlarged with GDP since it is the primary time series to be forecasted. However, the delay in the publication of many of these variables makes it difficult to assess the performance of economic activity in real time. To overcome this problem, and in line with Camacho and Perez Quiros (2010), alternative variables are further added to the estimation whenever the increase in the size of the data set raises the percentage of the variance of GDP explained by the common factor, but only when the variable to be added has (at least marginally) a statistically significant loading factor. Otherwise, the information provided by the potential indicator is assumed to be mainly idiosyncratic and it is not included in the model.

Following this principle, we extend the initial set of indicators in two dimensions. On the one hand, we include two hard indicators whose information has been crucial to assessing the economic developments of the global 2008-2009 recession: electricity consumption and registered unemployment. We additionally include consumer confidence and real credit card spending since they are early available indicators of internal demand (available with almost no publication delay). In the final specification of our model with all these indicators, the variance of GDP explained by the common factor is $71.4 \%$ and all the loading factors are statistically significant. ${ }^{13}$

Regarding the inclusion of financial indicators, we allow financial indicators to lead the business cycle dynamics in $h$ periods. To select the number of leads, we compute the log likelihood associated with lead times that go from one quarter to one and a half years. ${ }^{14}$ According to Fig. 1, which plots a summary of the highest log likelihood associated to different combinations of lead time periods for financial indicators, we find that the maximum of the likelihood function is achieved when the slope of the yield curve is allowed to lead the common factor by 9 months, and the rest of financial variables (credit, the spread and the mortgage rate minus 12-month Treasury bill rate) enter contemporaneously. ${ }^{15}$ In fact, this result goes in line with Wheelock and Wohar (2009) who find that the contemporaneous correlation between GDP growth and the slope of the yield curve is not statistically different from zero for the US, the UK and Germany, whereas the correlation with the slope lagged from one to six quarters are uniformly positive and statistically significant.

The estimated common factor and monthly estimates of quarterly GDP growth rates are plotted in Fig. 2. ${ }^{16}$ According to this figure, the evolution of the factor is in clear concordance with GDP growth and contains relevant information of its expansions

\footnotetext{
12 All the dynamic factor models use $p 1=6$ and $p 2=p 3=2$.

13 We tried with other real activity indicators such as Overnight Stays, Total Sales of Large Firms, Retail Sales, Imports, Exports and Euro-area GDP. However, in all of these cases we obtained that the percentage of the Spanish GDP that were explained by the model fell.

14 For purposes of simplicity, we have restricted Fig. 1 to include up to 12 leads only.

15 This also agrees with the results obtained from Table 3.

16 Recall that for those months where GDP is known, the actual values and the estimates of GDP coincide.
} 


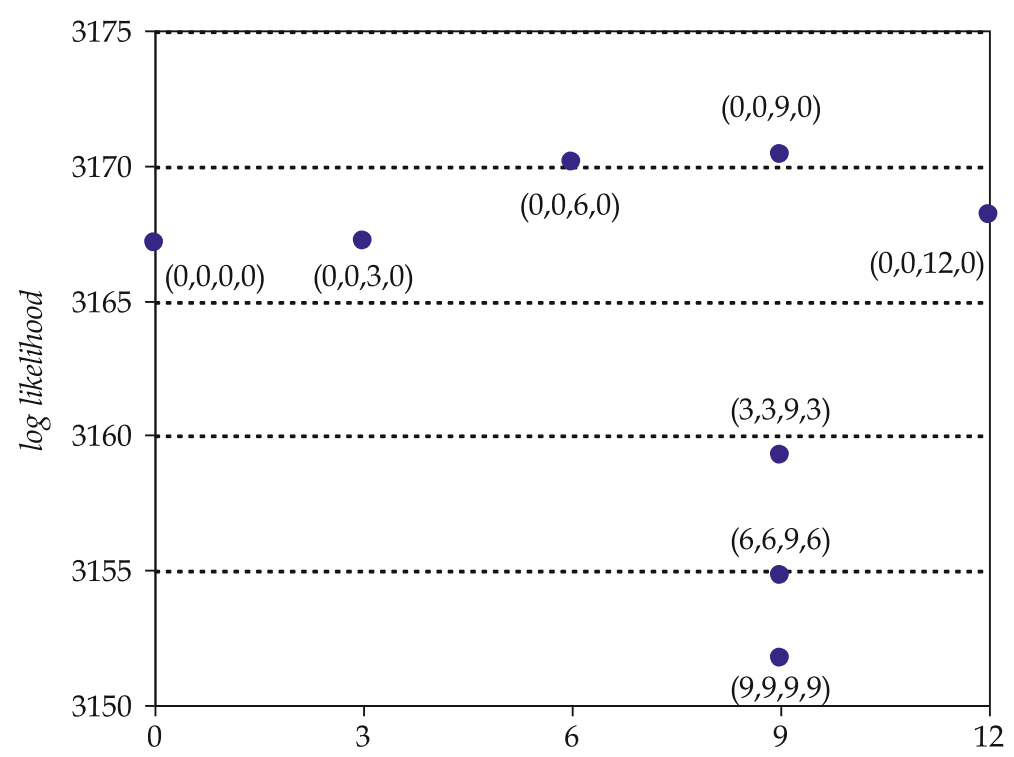

Fig. 1 Financial indicators at time $t$ have been related to the common factor at time $t+h$. In this figure, the value of $h$ for the slope of the yield curve appears on the horizontal axis and the log likelihood on the vertical axis. Numbers in brackets refer to the values of $h$ for the four financial variables in the following order: (1) credit, (2) spread, (3) slope and (4) the mortgage rate minus 12-month Treasury bill rate

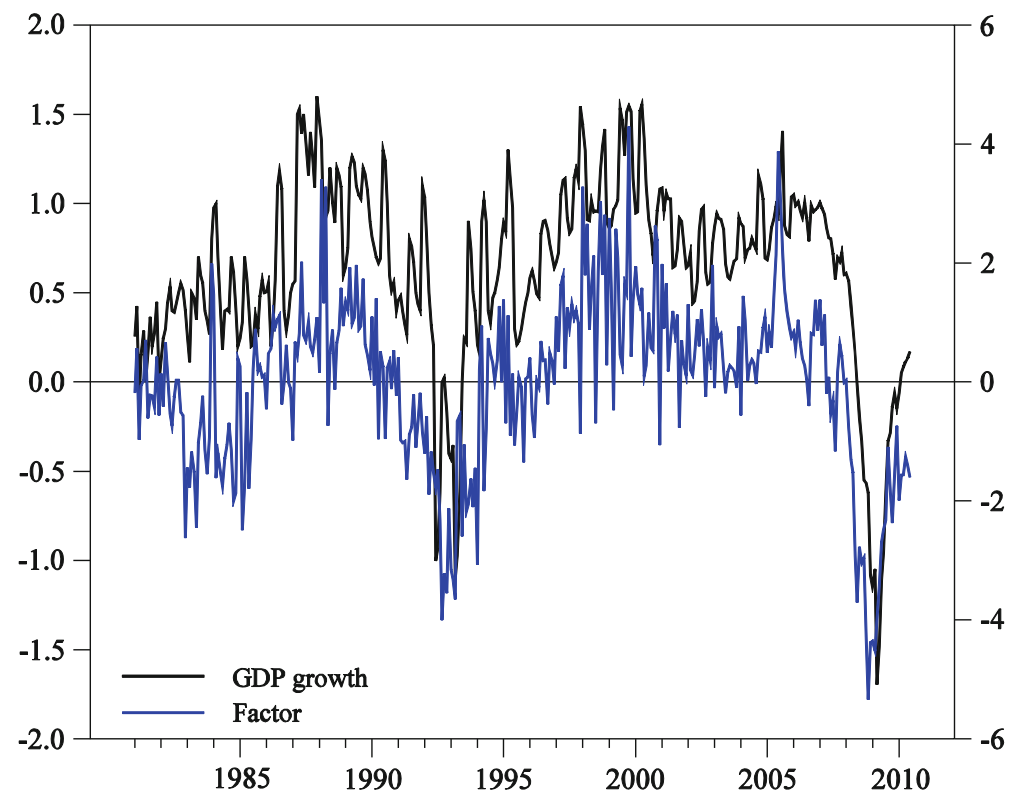

Fig. 2 Common factor and GDP growth. Notes The series are estimated from $12 \mathrm{~m} 1980$ to $12 \mathrm{~m} 2009$ 


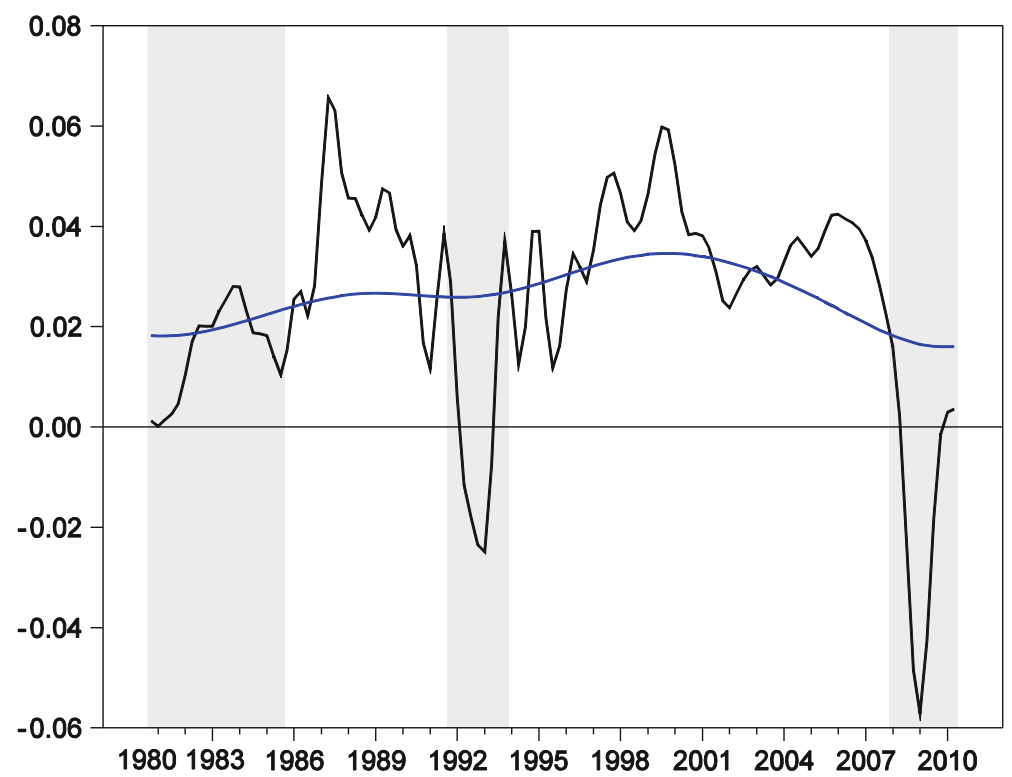

Fig. 3 Business cycle analysis. Notes Quarterly growth rates, in annual terms and potential growth. Shaded areas represent recessions as defined by ECRI

and recessions. ${ }^{17}$ Until the mid eighties, the Spanish GDP grew at reduced rates; this slowdown is explained by the negative values of the common factor. In 1986, Spain joined the European Union, and this year marks the beginning of values in the common factor that ends with the recession of 1993. In 1994 the recovery materializes and since then, the indicator exhibits consistently positive values over a period of fourteen years, ending in $2008 .{ }^{18}$ It is worth noting that over the sample, never before has the fall in the common factor been as deep as under the 2008-2009 recession (Fig. 3).

To further examine the business cycle information that can be extracted from the common factor, Fig. 4 plots the factor, the quarterly growth rates of GDP in annual terms and the potential growth (using the Hodrick-Prescott filter) along with shaded areas that represent recessions as defined by ECRI. Clearly, those periods that are marked by large slowdowns in the factor coincide with periods when GDP growth has been clearly below its potential and when ECRI has identified recessions in the Spanish economy. ${ }^{19}$

The loading factors, whose estimates appear in Table 4 (standard errors in parentheses), allow us to evaluate the correlation between the common factor and each of the indicators used in the model. Apart from GDP (loading factor of 0.18), the economic indicators with larger loading factors are hard and soft indicators. As expected, the

\footnotetext{
17 See Doménech and Gomez (2005), Doménech, Estrada and González (2007), and the references therein for an analysis of Spanish business cycles.

18 One noticeable exception is the potential short-lived decline in 1996.

19 ECRI dates the peaks in 80.03, 91.11, and 08.02, and the troughs in 85.05 and 93.11.
} 


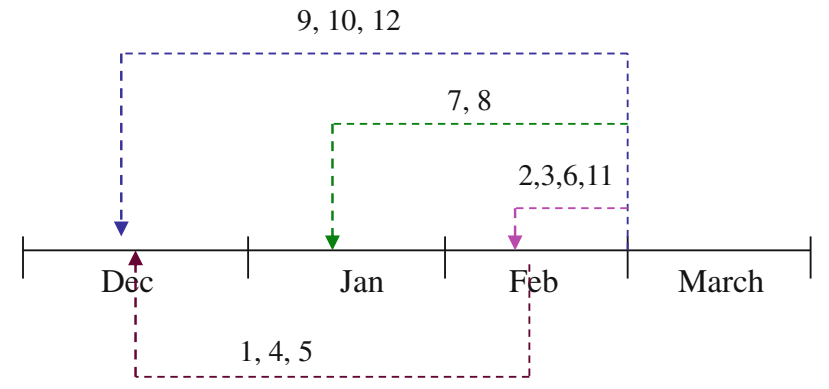

Fig. 4 Stylized real time data realizations. Notes The graph shows a stylized schedule of data releases. See Table 1 for associating the numbers with each indicator

Table 4 Loading factors

\begin{tabular}{|c|c|c|c|c|c|c|c|c|c|c|c|}
\hline GDP & $\mathrm{CCS}$ & $\mathrm{CC}$ & $\mathrm{EC}$ & RWI & IC & $\mathrm{U}$ & SSA & RCPS & MR12S & SLOPE & MR12TBR \\
\hline $\begin{array}{l}0.185 \\
(9.8)\end{array}$ & $\begin{array}{l}0.038 \\
(2.5)\end{array}$ & $\begin{array}{l}0.037 \\
(3.6)\end{array}$ & $\begin{array}{l}0.040 \\
(4.1)\end{array}$ & $\begin{array}{l}0.045 \\
(13.4)\end{array}$ & $\begin{array}{l}0.050 \\
(5.7)\end{array}$ & $\begin{array}{l}-0.014 \\
(3.2)\end{array}$ & $\begin{array}{l}0.064 \\
(27.6)\end{array}$ & $\begin{array}{l}0.019 \\
(3.9)\end{array}$ & $\begin{array}{l}-0.018 \\
(2.3)\end{array}$ & $\begin{array}{l}0.022 \\
(2.3)\end{array}$ & $\begin{array}{l}-0.024 \\
(2.3)\end{array}$ \\
\hline
\end{tabular}

Factor loadings ( $t$-ratios are in parentheses) measure the correlation between the common factor and each of the indicators appearing in columns. See Table 2 for a description of the indicators

loading factors for all of these indicators but unemployment are positive, indicating that these series are procyclical, i.e., positively correlated with the common factor.

Financial indicators exhibit significant correlations with the latent common factor. The correlation of the slope of the yield curve (with a lag of 3 quarters) with the current values of the common factor is positive so the more steeply sloped the yield curve, the higher the value of the common factor in the future. According to the loading factors estimates, the correlations of the two measures of financial markets tensions, the average mortgage rate minus the 12-month Euribor and the average mortgage rate minus the 12-month Treasury bill rate, are negative and statistically significant. Finally, the correlation of real credit to the private sector and the factor is positive.

\subsection{Simulated real-time analysis}

In real time, data are subject to important differences in publication lags which impose forecasters to compute their forecasts from unbalanced sets. Accordingly, we need to examine the forecast performance under the staggered release of monthly information, as it occurs in real time, so we do take account of publication lags in the data when computing the forecasts. Then, our forecast evaluation exercise is designed to replicate the typical situation in which the model is used with real-time data. ${ }^{20}$ For this purpose, we construct a sequence of data vintages from the final vintage data set similar to the pure real-time vintages, in the sense that delays in publications are incorporated. Since

\footnotetext{
20 In the simulated real-time analysis we take into account the real-time data flow and the recursive estimation of the model, without considering data revision. Due to data availability, pure real-time analyses are left for further research.
} 
we wanted to forecast GDP growth for almost 20 years from 1990.1 to 2009.1, the first data vintage of this experiment refers to data up to 1989.01 as it would be known on 15 June $1989 .{ }^{21}$ The vintages are then updated on the first day and on the fifteenth day of each month up to 1 July 2009, leading to 478 different vintages.

Because the data is released in blocks and the releases follow a relatively stable calendar, each forecast is conditional on the same (updated) set of data releases following the stylized schedule depicted in Fig. 4. If the data vintage is updated at the beginning of the respective month, the data set is updated with Credit card expenses, Consumer and Industry confidence indicators and yield-curve spread which are published with no delay, with Unemployment and Social Security affiliation which are assumed to be available with a 1-month delay, and with Credit, Mortgage and Stress, which are delayed 2 months. If the data vintage is updated at the middle of the month, the data set is enlarged with Income and Electricity demand, which appear with a delay of 1.5 months. In addition, at the middle of February, May, August and November, the data vintages are enlarged with the publication of the GDP series, which is assumed to be available with a delay of 1.5 months.

The way we treat real credit card spending $(C C S)$ and the slope of yield curve (SLOPE) should also be addressed. Although these variables are available on a daily basis and since the bulk of our data is monthly, to facilitate comparisons we follow the line suggested by Giannone et al. (2008). According to these authors, we disregard information from daily variables at frequencies lower than a month and let them enter the model as known at the end of the month.

Using the generated sequence of data vintages, the forecast simulations are carried out in a recursive way. With every new vintage, the dynamic factor model is re-estimated with the extended data set, and the nine-month blocks of forecasts are computed. According to this forecasting scheme, we consider series of forecasts for GDP growth in a certain quarter obtained in nine consecutive months. To understand how the forecasting exercise is developed in real time, Fig. 5 shows an example of a typical forecasting period. The forecasting period of GDP 1Q2009 starts with the first forecast computed on 08/15/08 and ends with the last backcast computed on 05/15/09. On $08 / 15 / 08$, the GDP for the second quarter of 2008 is known, so the model is reestimated with the corresponding data vintage and nine-month-ahead predictions are computed. For all the vintages issued from $08 / 15 / 08$ to $11 / 15 / 08$, the prediction procedure computes backcasts of the third quarter of 2008, nowcasts for the fourth quarter of 2008 and forecasts for the first quarter of 2009. On 11/15/08, and coinciding with the publication of the GDP figures for 3Q2008, the rolling window of 9 months forecasts is moved forward by computing backcasts for 4Q2008, nowcasts for 1Q2009 and forecasts for 2Q2009. The procedure is then repeated recursively until the last data vintage which refers to data obtained on 01/07/09.

This forecasting exercise allows us to asses the relative importance of forecasting from updated information sets. For example, according to Fig. 5, GDP predictions for the first quarter of 2009 are computed from forecasts (from 08/15/08 to 11/15/08), nowcasts (from 11/15/08 to 02/15/09) and backcasts (from 02/15/09 to 05/15/09).

\footnotetext{
21 According to the nine-month blocks of forecasts computed from the model, the first day on which the model produces forecasts of 1990.01 is June 15, 1989.
} 


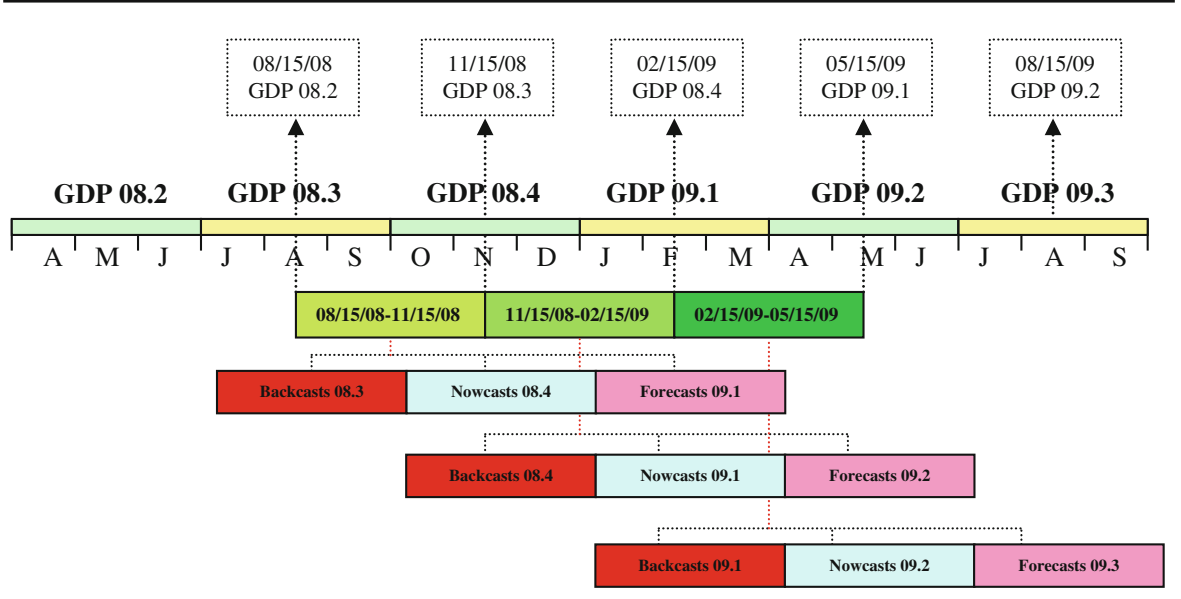

Fig. 5 Example of predictions. Notes Data release and the structure of different forecasts for GDP growth

Plots of actual data and real-time predictions can be found in Fig. 6. This figure shows the simulated real-time predictions (straight lines) of Spanish GDP as well as the corresponding final quarterly data (dashed lines). Hence, panels 1, 2 and 3 in Fig. 6 correspond to backcasts, nowcasts and forecasts (updated each fifteen days) of the same actual values of GDP growth which are equally distributed among the respective days of the quarter to facilitate comparisons. Accordingly, these charts differ from each other in the information sets used on the day that the predictions were computed.

Several noteworthy features of Fig. 6 stand out. First, overall the series of actual releases and real-time forecasts possess a high degree of conformity. Forecasts follow sequential patterns that track the business cycle marked by the evolution of GDP releases. Second, the real-time estimates become more accurate in the case of backcasts since the predictions are computed immediately before the end of the quarter using larger information sets. In many cases, there is very little difference between the value for actual GDP and the real-time estimate immediately prior to the release. Third, nowcasts and forecasts track the GDP dynamics with some delays since they use poorer information sets to compute predictions although they are available sooner.

Table 5 shows the mean-squared forecast errors (MSE), which are the average of the deviations of the predictions from the final releases of GDP available in the data set. Results for backcasts, nowcasts and forecasts appear in the second, third and fourth columns of the table, respectively. In addition to the factor model, two benchmark models are included in the forecast evaluation. The former is an autoregressive model of order two which is estimated in real-time producing iterative forecasts, and the latter is a random walk model whose forecasts are equal to the average of the latest available real-time observations. The immediate conclusion obtained when comparing the forecasts is that it is beneficial to use the dynamic factor model in forecasting the Spanish GDP in terms of the forecast horizon. The differences between the MSE results using the factor model and the benchmark models are noticeable and range from relative MSE of 0.39 to 0.84 .

The table also includes the within recessions and within expansions MSE, which are computed from the periods that have been identified by ECRI as recessions and 

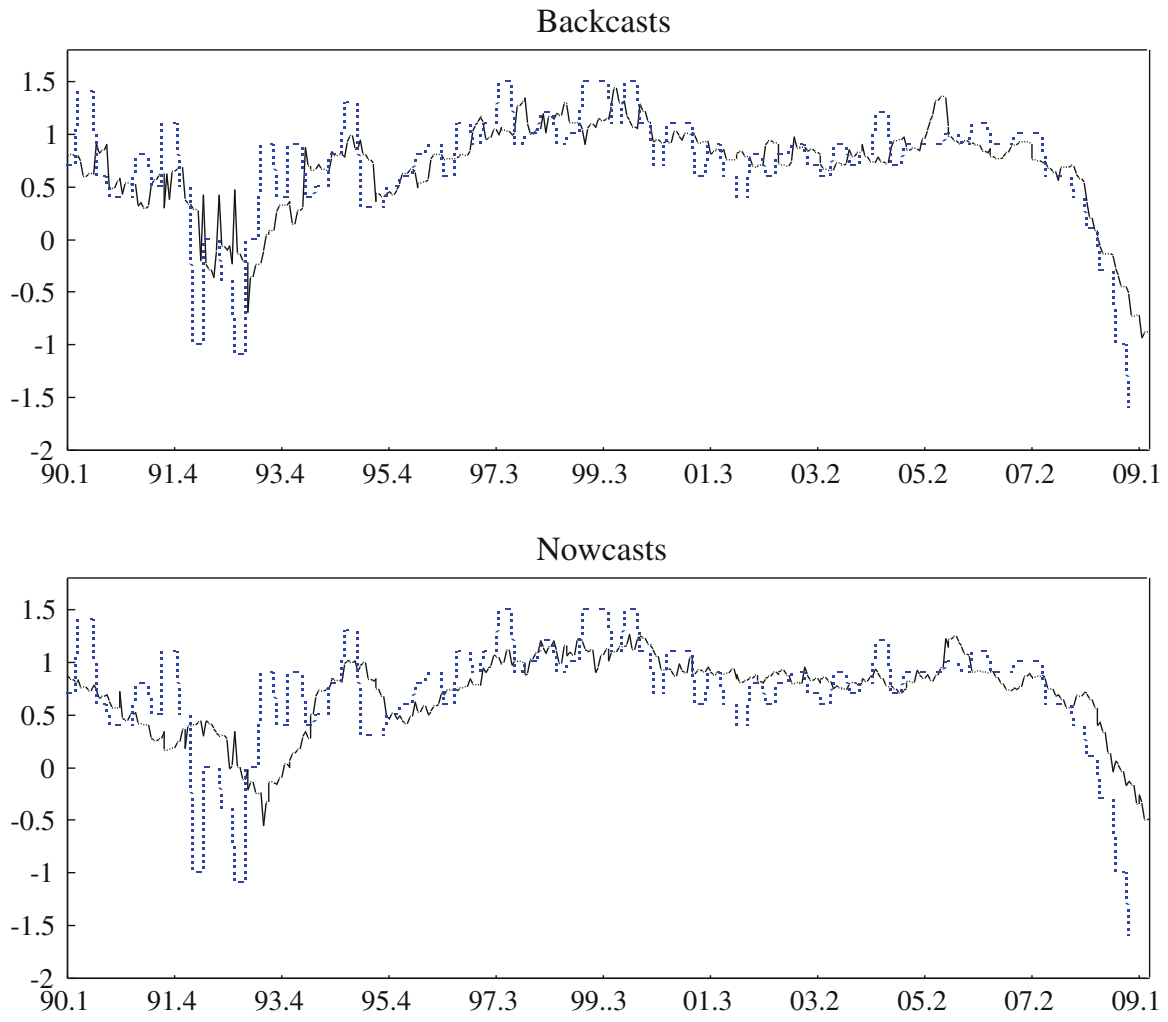

Forecasts

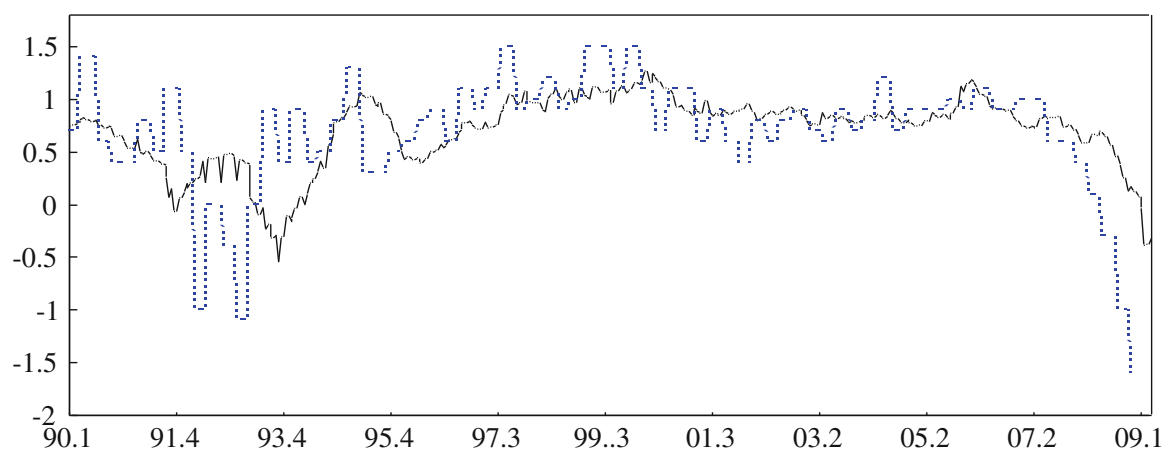

Fig. 6 Real time predictions and actual realizations. Notes Actual realizations of GDP growth (dotted line) and real time predictions, backcasts (top), nowcasts (middle) and forecasts (bottom panel)

expansions. The figures of the table show that the forecasting accuracy of the models varies considerably over the business cycle. In recessions, although there is a marked deterioration for all models, the relative loss in forecasting accuracy from the benchmark models is magnified with respect to that in the expansionary periods. The intuition is that the evolution of GDP in expansions is quite flat around its historical 
Table 5 Predictive accuracy

\begin{tabular}{|c|c|c|c|c|c|c|}
\hline & Back & & Now & & Fore & \\
\hline \multirow[t]{2}{*}{ MSE-MICA } & 0.138 & & 0.194 & & 0.260 & \\
\hline & $\mathrm{R}: 0.470$ & E: 0.069 & R: 0.795 & E: 0.070 & R: 1.078 & E: 0.090 \\
\hline \multirow[t]{2}{*}{ MSE-RW } & 0.351 & & 0.357 & & 0.361 & \\
\hline & $\mathrm{R}: 1.434$ & E: 0.131 & $\mathrm{R}: 1.457$ & E: 0.133 & R: 1.469 & E: 0.135 \\
\hline MSE-MICA/MSE-RW & 0.392 & & 0.543 & & 0.720 & \\
\hline \multirow[t]{2}{*}{ MSE-AR } & 0.207 & & 0.280 & & 0.309 & \\
\hline & R: 0.808 & E: 0.085 & R: 1.193 & E: 0.095 & $\mathrm{R}: 1.303$ & E: 0.108 \\
\hline MSE-MICA/MSE-AR & 0.665 & & 0.692 & & 0.840 & \\
\hline MSE-MICA2 & 0.137 & & 0.202 & & 0.303 & \\
\hline MSE-MICA/MSE-MICA2 & 1.004 & & 0.950 & & 0.847 & \\
\hline \multicolumn{7}{|c|}{ Equal predictive accuracy tests } \\
\hline DM-RW & 0.0001 & 0.0004 & 0.0046 & & & \\
\hline DM-AR & 0.0002 & 0.0008 & 0.0581 & & & \\
\hline DM-MICA2 & 0.7523 & 0.5383 & 0.0449 & & & \\
\hline MDM-RW & 0.0001 & 0.0004 & 0.0049 & & & \\
\hline MDM-AR & 0.0002 & 0.0009 & 0.059 & & & \\
\hline DM-MICA2 & 0.7527 & 0.5391 & 0.0457 & & & \\
\hline WSR-RW & 0.0000 & 0.0000 & 0.0000 & & & \\
\hline WSR-AR & 0.0000 & 0.0000 & 0.0000 & & & \\
\hline WSR-MICA2 & 0.5818 & 0.0419 & 0.0115 & & & \\
\hline MGN-RW & 0.0000 & 0.0000 & 0.0000 & & & \\
\hline MGN-AR & 0.0000 & 0.0000 & 0.0000 & & & \\
\hline MGN-MICA2 & 0.6810 & 0.0446 & 0.0000 & & & \\
\hline MR-RW & 0.0000 & 0.0000 & 0.0000 & & & \\
\hline MR-AR & 0.0000 & 0.0000 & 0.0000 & & & \\
\hline MR-MICA2 & 0.7348 & 0.0000 & 0.0000 & & & \\
\hline \multicolumn{7}{|l|}{ Encompassing tests } \\
\hline RW/MICA & 0.0000 & 0.0000 & 0.0000 & & & \\
\hline AR/MICA & 0.0000 & 0.0000 & 0.0000 & & & \\
\hline MICA2/MICA & 0.0011 & 0.0000 & 0.0003 & & & \\
\hline
\end{tabular}

The forecasting sample is 1Q1990-1Q2009, which implies comparisons over 478 forecasts. Entries in rows one to seven are Mean Squared Errors (MSE) of MICA, Random Walk (RW), autoregressive of order two (AR), and MICA that does not include finance variables (MICA2), and the relative MSEs over that of MICA. R and E refer to recessions and expansions periods according to ECRI. The next fifteen rows show the $p$-values of the following tests of equal forecast accuracy: DM (Diebold-Mariano), MDM (modified DM), Wilconson's Signed-Rank (WSR), MGN (Morgan-Granger-Newbold), and MR (Meese-Rogoff), all of them described in Diebold and Mariano (1995) and Harvey et al. (1997). The last three rows present the $p$-values of the forecast encompassing test which is based upon the significance test of $a_{1}$ in the OLS regression $y_{t}-\hat{y}_{t, i}=a_{0}+a_{1} \hat{y}_{t, M I C A}+\varepsilon_{t}$, where $\hat{y}_{t, M I C A}$ is the forecast from MICA and $\hat{y}_{t, i}$ is either the forecast from RW, AR, and MICA2

average. Therefore, according to the simulations developed in Sect. 2.5, the relative reductions obtained from the dynamic factor model diminish considerably.

The relative gains of using financial indicators in forecasting GDP is also examined in Table 5. For this purpose, the forecasting accuracy of a dynamic factor model that does not use financial indicators (labelled as MICA2) is also showed in the table. Although the difference in backcasting accuracy from MICA and MICA2 is not statistically significant, the relative gains from the model that uses the financial indicators increase with the forecasting horizon. In particular, the relative MSE is reduced to 0.95 
in nowcasting, and to 0.85 in forecasting and the last reductions become statistically significant. This result confirms the leading forecasting ability of financial indicators.

Note that the MSE leads to a ranking of the competing models according to their forecasting performance. However, it is advisable to test whether the forecasts made with the dynamic factor model are significantly superior to the others models' forecasts. One interesting possibility is to test the null hypothesis of no difference in the forecasting accuracy of these competing models. Among the extensive set of different tests proposed in the literature, Table 5 displays the results of the following tests: DM (Diebold-Mariano), MDM (modified DM), Wilconson's Signed-Rank (WSR), MGN (Morgan-Granger-Newbold), and MR (Meese-Rogoff), all of them described in Diebold and Mariano (1995) and Harvey et al. (1997). The last two rows in Table 4 present the $p$-values of the forecast encompassing test, which is based upon the significance test of the coefficient $a_{1}$ in the following OLS regression

$$
y_{t}-\hat{y}_{t, i}=a_{0}+a_{1} \hat{y}_{t, M I C A}+\varepsilon_{t}
$$

where $\hat{y}_{t, M I C A}$ is the forecast from MICA and $\hat{y}_{t, i}$ is either the forecast from RW and AR. $^{22}$

Noticeably, Table 5 shows that the $p$-values of the equal forecast accuracy tests computed for backcasts and nowcasts are always less than 0.05 , revealing that the dynamic factor model is statistically superior to the benchmark competitors. The results for forecasts are qualitatively similar to the case of backcasts and nowcasts with the exception of the comparison between MICA and AR models. In this case, one can reject the null hypothesis of equal forecast accuracy at significance level higher than 0.06 although this significance level is still quite small. Finally, the $p$-values of the equal forecast accuracy tests reject the null hypothesis that either AR or RW forecasts encompass MICA forecasts at all confidence levels.

\section{Conclusions}

This paper proposes an extension of the Stock and Watson (1991) single-index dynamic factor model and evaluates it for forecasting exercises of Spanish quarterly GDP growth. The model has the advantage of combining information from real and financial indicators with different frequencies, short samples and publication lags. Using the Kalman filter, the model computes estimates of the unobserved common coincident component and of any missing values in the different series used to estimate the model.

Our results indicate three interesting features. First, we find that the common factor reflects the behavior of the Spanish GDP growth during expansions and contractions very well. Second, we show that financial indicators such as the slope of the yield curve and the growth rate of real credit are useful for forecasting output growth especially when assuming that some financial variables lead the common factor. Finally, we provide a simulated real-time exercise that is designed to replicate the data availability

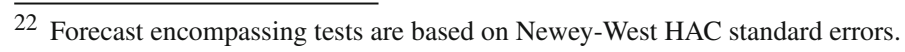


situation that would be faced in a true real-time application of the model. We show that the model is a valid tool to be used for short-term analysis.

The analysis in this paper highlights some lines for future research. First, although the model presented in this paper provides timely estimates of the state of real activity, it does not provide measures of the economic activity at frequencies higher than monthly. This is still a developing area but several ongoing studies such as Aruoba et al. (2009) are exploring this possibility. Second, although we examine the forecasting accuracy of the model by using a pseudo real-time exercise that accounts for recursive estimations and the typical delays observed in data publications, it uses final data vintages and, hence, ignores statistical revisions to earlier data releases. Although the actual data vintages that would have been used by real-time forecasters are hard to be obtained, we believe that allowing for such revisions is an interesting exercise for further assessing forecasting accuracy of our model in real-time.

Open Access This article is distributed under the terms of the Creative Commons Attribution License which permits any use, distribution and reproduction in any medium, provided the original author(s) and source are credited.

\section{References}

Alvarez R, Camacho M, Perez Quiros G (2011) Finite sample performance of small versus large scale dynamic factor models. Universidad de Murcia, Mimeo

Angelini E, Camba-Mendez G, Giannone D, Reichlin L, Runstler G (2011) Short-term forecasts of Euro area GDP growth. Econom J 14:C25-C44

Aruoba B, Diebold F, Scotti C (2009) Real-time measurement of business conditions. J Bus Econ Stat 7:417-427

Camacho M, Sancho I (2003) Spanish diffusion indexes. Span Econ Rev 5:173-203

Camacho M, Perez Quiros G (2010) Introducing the Euro-STING: short term INdicator of euro area growth. J Appl Econom 25:663-694

Camacho M, Perez Quiros G (2011) Spain-STING: Spain short term INdicator of growth. Manch Sch 79:594-616

Cuevas A, Quilis E (2009) A factor analysis for the Spanish economy (FASE). Mimemo, Ministerio de Economía y Hacienda, Madrid

Diebold F, Mariano R (1995) Comparing predictive accuracy. J Bus Econ Stat 13:253-263

Doménech R, Gomez V (2005) Ciclo economico y desempleo estructural en la economia española. Investig Econ 19:259-288

Doménech R, Estrada González-Calvet L (2007) Potential growth and business cycle in the Spanish economy: implications for fiscal policy. Mimeo. Universidad de Valencia Working Paper 0705

Giannone D, Reichlin L, Small D (2008) Nowcasting: the real-time informational content of macroeconomic data. J Monet Econ 55:665-676

Gomez V, Maravall A (1996) Programs TRAMO (Time series Regression with Arima noise, Missing observations, and Outliers) and SEATS (Signal Extraction in Arima Time Series). Instructions for the user. Working paper 9628, Research Department, Banco de España

Harvey D, Leybourne S, Newbold P (1997) Testing equality of prediction mean squared errors. Int J Forecast $13: 287-291$

Herce J (2004) Las fuentes de crecimiento de la economía española entre 1960 y 2003. FEDEA working paper

Mariano R, Murasawa Y (2003) A new coincident index of business cycles based on monthly and quarterly series. J Appl Econom 18:427-443

Proietti T, Moauro F (2006) Dynamic factor analysis with non linear temporal aggregation constraints. Appl Stat 55:281-300 
Stock J, Watson M (1991) A probability model of the coincident economic indicators. In: Lahiri K, Moore $\mathrm{G}$ (eds.) Leading economic indicators, new approaches and forecasting records. Cambridge University Press, Cambridge

Stock J, Watson M (2002) Macroeconomic forecasting using diffusion indexes. J Bus Econ Stat 20:147-162 Wheelock D, Wohar M (2009) Can the term spread predict output growth and recessions? A survey of the literature. Fed Reserve Bank St. Louis Rev 91:419-440 